\title{
What is the origin of the mass of the Higgs boson?
}

\author{
M. Novello壮 and E. Bittencourt团 \\ Instituto de Cosmologia Relatividade Astrofisica ICRA - CBPF \\ Rua Dr. Xavier Sigaud, 150, CEP 22290-180, Rio de Janeiro, Brazil
}

(Dated: May 22, 2018)

\begin{abstract}
The purpose of this paper is to present a unified description of mass generation mechanisms that have been investigated so far and that are called the Mach and Higgs proposals. In our mechanism, gravity acts merely as a catalyst and the final expression of the mass depends neither on the intensity nor on the particular properties of the gravitational field. We shall see that these two strategies to provide mass for all bodies that operate independently and competitively can be combined into a single unified theoretical framework. As a consequence of this new formulation we are able to present an answer to the question: what is the origin of the mass of the Higgs boson?
\end{abstract}

PACS numbers: $98.80 . \mathrm{Cq}$

\section{INTRODUCTION}

In order to become a reliable candidate as a mechanism to generate mass, there are three indispensable conditions that such mechanism has to fulfil, to wit:

- There must exist a universal field that interacts with all kinds of particles;

- This field must be such that its interaction with matter breaks explicitly some symmetry that only massless particles exhibit, e.g. the gauge freedom for vector fields or the chirality for fermions;

- There must exist a free parameter such that different bodies can acquire distinct values for their corresponding mass (the spectrum of mass).

There are only two fashionable candidates that fulfill the first condition:

- The gravitational field;

- A scalar field $\varphi$.

The Higgs boson $\varphi$ was postulated to couple universally with all kinds of matter. The other candidate, gravity, is known to couple with all forms of matter and energy and its universality is recognized as a scientific truth. We note that after accepting either one of these two fields as a good candidate that fulfills the first requirement, it is not a hard job to elaborate scenarios such that the other two conditions are satisfied too. We would like to compare these two mechanisms and analyze the conditions under which their strategies can be combined.

In order to simplify our analysis we will overview the generation of mass for spinor fields. The generalization for bosons is made along the same lines. In both cases

\footnotetext{
${ }^{*}$ M. Novello is Cesare Lattes ICRANet Professor

${ }^{\dagger}$ Electronic address: novello@cbpf.br

${ }^{\ddagger}$ Electronic address: eduhsb@cbpf.br
}

the origin of the mass of any body $\mathbb{A}$ depends on its interaction with its surroundings yielding an overall effect (described either as a scalar field - in the case of the Higgs mechanism - or as the metric tensor of the geometry of space-time, in the case of the gravitational origin) on $\mathbb{A}$ which is represented by a distribution of energy given by the form

$$
T_{\mu \nu}=\Lambda g_{\mu \nu}
$$

In the literature concerning General Relativity this form of energy-momentum tensor is attributed to the cosmological constant introduced by Einstein in order to be able to construct a model for the geometry of the Universe. In the realm of quantum field theory, such distribution is identified as the vacuum. It is true that if one considers the Machian point of view that the inertia of a body $\mathbb{A}$ depends on the energy distribution of all others bodies in the Universe, then $\Lambda$ should be interpreted as the cosmological constant. However this is not mandatory. The term rest-of-the-universe concerns the environment of $\mathbb{A}$, that is the whole domain of influence on $\mathbb{A}$ of the remaining bodies in the Universe.

The idea of using a scalar field to be at the origin of the mass appeared in the domain of high energy physics and it received the name "Higgs mechanism" [1]. On the other hand, the relationship of mass with gravity is a very old one. Such deep connection has been emphasized in a qualitative way many times.

According to Mach, 2] inertia is related to the global distribution of energy of all particles in the Universe. From a historical point of view, this idea led Einstein to the development of a new theory of gravitation. However, the dependence of inertia on global structures of the Universe was lost.

Otherwise, a mechanism of mass generation came from microphysics. Indeed, the Higgs model produced an efficient scenario for generating mass to all bodies which goes in the opposite direction of Mach's proposal. In this mechanism, a global symmetry transforms into a local one in the presence of vector gauge fields. Then, a self-interaction term of an associated scalar field in its fundamental state, represented by $T_{\mu \nu}=L_{\text {int }}\left(\phi_{0}\right) g_{\mu \nu}$, 
appears as the vehicle which provides mass to the gauge field.

Recently a new mechanism for generation of mass that is a realization of Mach's idea was proposed [3]. The main idea of this new proposal is to couple nonminimally the field under consideration to gravity through the space-time curvature. The vacuum energy distribution of the rest-of-the-universe is represented by the cosmological term $\Lambda$. In the realm of General Relativity the dynamics of the metric of the space-time together with the $\Lambda$-term is precisely responsible to generate mass to the field.

The great novelty of this mechanism is that the gravitational field acts merely as a catalyst, once the final expression of the mass depends neither on the intensity nor on the particular properties of the gravitational field. It was precisely the wrong belief that the value of the mass obtained through any gravitational scheme should depend on the properties of the gravitational field that was responsible for not considering gravity as an important actor in the mechanism of generating mass. We review briefly the alternative Higgs mechanism in order to compare both processes.

\section{THE MASS OF FERMIONS}

A. The Higgs vacuum $T_{\mu \nu}=\Lambda g_{\mu \nu}$

Consider a theory of a real scalar field $\varphi$ described by the Lagrangian

$$
\mathbb{L}=\frac{1}{2} \partial_{\mu} \varphi \partial^{\mu} \varphi-V(\varphi)
$$

where the potential has the form

$$
V=\frac{1}{2} \mu^{2} \varphi^{2}+\frac{\lambda}{4} \varphi^{4}
$$

In the homogeneous case, in order to satisfy the equation of motion, the field must be in an extremum of the potential, which is true for two classes of solution: either

$$
\varphi=0
$$

or

$$
\varphi_{0}^{2}=-\frac{\mu^{2}}{\lambda}
$$

In order to be a minimum the constant $\mu^{2}$ must be negative. This is a problem, since it should imply that the mass of the scalar field is imaginary. This difficulty is in general avoided in the following manner. One starts by redefining the field by the introduction of a new real variable $\chi$ :

$$
\varphi=\varphi_{0}+\chi
$$

where $\varphi_{0}$ is a constant. Substituting this definition on Lagrangian (2), it follows that

$$
\mathbb{L}=\frac{1}{2} \partial_{\mu} \chi \partial^{\mu} \chi+\mu^{2} \chi^{2}-\frac{\lambda}{4} \chi^{4}-\lambda \varphi_{0} \chi^{3}+\frac{\mu^{4}}{4 \lambda} .
$$

This Lagrangian represents a real scalar field $\chi$ with real positive mass $m^{2}=-\mu^{2}$ and extra terms of selfinteraction. Note that in the Lagrangian it appears a residual constant term representing a background constant negative energy distribution

$$
T_{\mu \nu}(\text { residual })=-\frac{\mu^{4}}{4 \lambda} g_{\mu \nu}
$$

In the realm of high-energy physics it is considered that such a term "... has no physical consequences and can be dropped" [4]. We will come back to this when we analyze its gravitational effects.

Note that now, the potential of field $\chi$ takes the form

$$
V=m^{2} \chi^{2}+\frac{\lambda}{4} \chi^{4}+\lambda \varphi_{0} \chi^{3}
$$

Its minimum occurs for $\chi=0$, which is the point around which the expansion of the field must be made. Let us couple this scalar field with a spinor $\Psi$ through the Lagrangian

$$
\mathbb{L}=\frac{1}{2} \partial_{\mu} \varphi \partial^{\mu} \varphi-V(\varphi)+\mathbb{L}_{D}+L_{i n t}
$$

where $\mathbb{L}_{D}$ is Dirac dynamics for massless free field. The interaction term is $L_{\text {int }}=-\xi \varphi F(\Phi)$, where we define $\Phi \equiv \bar{\Psi} \Psi$. Expanding in power series with respect to $\Phi$ and keeping the first order, as usual, we set this term as $-\xi \varphi \Phi=-\xi \varphi \bar{\Psi} \Psi$. Making the same replacement that we previously made using $\chi$, instead of $\varphi$, this theory becomes

$$
\mathbb{L}=\frac{1}{2} \partial_{\mu} \chi \partial^{\mu} \chi-V(\chi)+\mathbb{L}_{D}-\xi\left(\varphi_{0}+\chi\right) \bar{\Psi} \Psi .
$$

The equation for the spinor field becomes

$$
i \gamma^{\mu} \partial_{\mu} \Psi-\xi \varphi_{0} \Psi-\xi \chi \bar{\Psi} \Psi=0
$$

which represents a spinor field of mass $\xi \varphi_{0}>0$ interacting with a scalar field $\chi$.

\section{B. The gravitational vacuum $T_{\mu \nu}=\Lambda g_{\mu \nu}$}

We restrict our analysis here only to the case of fermions. The massless theory for a spinor field is given by Dirac equation

$$
i \gamma^{\mu} \partial_{\mu} \Psi=0
$$

This equation is invariant under $\gamma^{5}$ transformation. In order to have mass for the fermion this symmetry must be broken. Who is the responsible for this? 


\section{Gravity breaks the symmetry}

In the generation of mass through the mechanism that we are analyzing here, gravity is responsible for breaking the symmetry. In order to understand this we have to face the following question: how gravity interacts with fermions? Many authors argue that the minimal coupling principle drives this interaction. However there are others principles involved in the choice of the form that gravity acts on matter. Let us take for instance the example of the scalar field. Following the paradigm of the electromagnetic field, it is generally accepted that the dynamics of massless fields should be invariant under conformal transformations. In the case of scalar field this can be achieved only by taking into account a direct coupling with the curvature of space-time. In other words, the dynamics of the scalar field in the presence of gravity acquires a term of the form

$$
L_{i n t}=\frac{1}{6} R \varphi^{2}
$$

Indeed, the presence of this extra term is sufficient to yield the conformal invariance of the dynamics of the scalar field. We have presented this example here only to remind the reader that the status of the minimal coupling principle to drive the dynamics of the interaction of any field with gravity is not a imperative law that should be followed in any circumstance but only a suggestion that happens to be valid for tests particle. It cannot be transformed in a paradigm for the behavior of arbitrary fields under gravitational interaction.

Thus, in the framework of General Relativity we set for the gravitational interaction of the fermion the following Lagrangian

$$
\begin{aligned}
\mathbb{L} & =\frac{i}{2} \bar{\Psi} \gamma^{\mu} \nabla_{\mu} \Psi-\frac{i}{2} \nabla_{\mu} \bar{\Psi} \gamma^{\mu} \Psi \\
& +\frac{1}{\kappa}\left(1+\frac{\sigma}{4} \Phi\right)^{-2} R-\frac{1}{\kappa} \Lambda \\
& -\frac{3}{\kappa} \frac{\sigma^{2}}{8}\left(1+\frac{\sigma}{4} \Phi\right)^{-4} \partial_{\mu} \Phi \partial^{\mu} \Phi
\end{aligned}
$$

where $\sigma$ is a constant coupling. The first two terms of $\mathbb{L}$ represents the free part of the spinor field. The next term represents the nonminimal coupling interaction of $\Psi$ with gravity. The vacuum - represented by $\Lambda$-is added by the reasons presented above and it must be understood as the definition of expressing the influence of the restof-the-universe on $\Psi$. The last term of the Lagrangian is responsible to avoid higher-order derivatives of the spinor field

Using the equations of motion obtained by varying both the metric tensor $g_{\mu \nu}$ and the spinor field $\Psi$ and after some algebraic manipulations the equation for $\Psi$ becomes

$$
i \gamma^{\mu} \nabla_{\mu} \Psi-M \Psi=0
$$

where

$$
M=\frac{\sigma \Lambda}{\kappa c^{2}}
$$

Thus, as a result of the coupling of the spinor field with gravity the spinor field acquires a mass $M$ that depends crucially on the existence of $\Lambda$. If $\Lambda$ vanishes, then the mass of the field vanishes. Let us note that there is another interpretation of the Lagrangian (8) that is worth pointing out here. Let us define the nondimensional scalar field $\eta$ by setting

$$
\eta=\frac{1}{1+\frac{\sigma}{4} \Phi}
$$

Then, in terms of this new quantity the dynamics can be rewritten as

$$
\mathbb{L}=\mathbb{L}_{D}-\frac{\Lambda}{\kappa}-\frac{6}{\kappa}\left(\partial_{\mu} \eta \partial^{\mu} \eta-\frac{1}{6} R \eta^{2}\right)
$$

which is nothing but the equation of a scalar field $\eta$ conformally coupled to the space-time curvature.

\section{NUMERICAL RESULTS}

Let us point out some of the observational consequences of such mechanism. We start by recalling that the inverse Compton length of any particle is given in terms of its mass $M$, the Planck constant $\hbar$ and light velocity $c$ yielding

$$
\mu=\frac{c}{\hbar} M
$$

For later use, we rewrite it in terms of gravitational quantities using the Newton constant $G_{N}$ or, equivalently, the Einstein constant $\kappa$. The Schwarzschild solution of the gravitational field of a static compact object has an horizon - that is a one-way membrane - characterized by its Schwarzschild radius

$$
r_{s}=\frac{1}{4 \pi} \kappa M c^{2} \text {. }
$$

Using the definition of the Planck length

$$
L_{P l}^{2} \equiv \frac{1}{8 \pi} \kappa \hbar c
$$

it follows that the inverse Compton length may be written under an equivalent form as the ratio between the corresponding Schwarzschild radius and the Planck length squared:

$$
\mu=\frac{1}{2} \frac{r_{s}}{L_{P l}^{2}} .
$$

The formula of the mass, obtained in Eq. (10) from the nonminimal coupling of a spinor field $\Psi$ with gravity, is expressed in terms of the cosmological constant $\Lambda$, 
the Planck length and parameter $\sigma$ of the nonminimal coupling yielding the expression for the inverse Compton wavelength

$$
\mu=\frac{1}{8 \pi} \frac{\sigma \Lambda}{L_{P l}^{2}}, \quad \Longrightarrow \quad M=\frac{\hbar}{8 \pi c} \frac{\sigma \Lambda}{L_{P l}^{2}} .
$$

This expression relates two parameters: the mass $M$ and the associated nonminimal coupling constant with gravity $\sigma$ that has the dimensionality of volume. The knowledge of one of these two parameters ( $M$ or $\sigma$ ) allows the knowledge of its companion. By comparison of the above two expressions of $\mu$, that is, Compton definition Eq. (12) and our formula for the mass Eq. (13) yields the expression of $\sigma$ :

$$
\sigma=4 \pi \frac{r_{s}}{\Lambda}
$$

Thus, different fermionic particles that have different masses have different values of $\sigma$. We note furthermore that the ratio $M / \sigma$ which has the meaning of a density of mass is a universal constant given only in terms of $\kappa$ and $\Lambda$. How to interpret such universality? There is a direct and simple way that is the following: we rewrite this formula as a density of energy, that is

$$
\frac{M c^{2}}{\sigma}=\frac{\Lambda}{\kappa} .
$$

The right-hand side is nothing but the density of energy of the vacuum. Thus we can say that $\sigma$ is the volume in which an homogeneous distribution of the particle energy spreads having the same value of the vacuum energy density provided by the cosmological constant, that is, $\Lambda / \kappa$.

Once our formula of mass for fermions contains gravitational quantities which are well known to be extremely small, let us compare it with actual numbers that we can get, for instance, from the simplest example of the electron. The main question is: should the coupling constant $\sigma$ become an enormously big value in order to compensate the weakness of the gravitational field? A direct calculation for the known elementary particles show that this is not the case. This is a direct consequence of the fact that in the process of giving mass gravity enters only as a catalyst. Indeed, for the simple stable lepton, the electron $e^{-}$, we find that its gravitational horizon is given by

$$
r_{s} \approx 10^{-55} \mathrm{~cm}
$$

which implies that

$$
\sigma_{e} \approx 125 \mathrm{~cm}^{3}
$$

The substance that we call the electron is tremendously concentrated within its Compton wavelength $\lambda_{c}$. Indeed if we compare the density of energy $M_{e} c^{2} / r^{3}$ for $\lambda_{c}^{3}$ and $\sigma$ it follows that all of the electron is concentrated in the interior of its Compton volume:

$$
\frac{\varrho_{c}}{\varrho_{\sigma}} \approx 10^{31}
$$

\section{MINIMAL MASS VALUE}

The present method of evaluating the mass takes into account only classical gravitational aspects. Thus, in principle it cannot be applied at the quantum level. Indeed, quantum effects become non-negligible at least at the Compton wavelength of a given particle. This means that there is a threshold of applicability of our mechanism. In other words the value of the length associated to the gravitational mechanism must be higher than the corresponding Compton wavelength of the particle. This led naturally to the minimum value of the mass of any fermion - called $M_{q}$ - that can be generated by the present gravitational procedure. This value is obtained by the condition

$$
M_{q} c^{2} \geq \frac{\Lambda}{\kappa} \frac{\hbar^{3}}{M_{q}^{3} c^{3}} .
$$

Inserting the current value of the constants that appear in the right-hand side it follows that the minimum possible value for the mass is

$$
M_{q} \geq 2.36 \times 10^{-3} \mathrm{eV} .
$$

In the gravitational procedure of generation of mass there is no possibility of having a fermion with a mass lower than $M_{q}$.

This procedure allows us to state that the mechanism proposed here is to be understood as a realization of the Mach principle according to which the inertia of a body depends on the background of the rest-of-the-universe. This strategy can be applied in a more general context in support of the idea that (local) properties of microphysics may depend on the (global) properties of the Universe. In the case $\sigma=0$, the Lagrangian reduces to a massless fermion satisfying Dirac's dynamics plus the gravitational field described by General Relativity.

\section{WHAT DOES GIVE MASS TO THE SCALAR FIELD THAT GIVES MASS FOR THE VECTOR AND SPINOR FIELDS?}

In the preceding section we described the Higgs model that produced an efficient scenario for generating mass in the realm of high-energy physics. This mechanism appeals to the intervention of a scalar field that is the vehicle which provides mass to the spinor field $\Psi$. For the mass to be a real and constant value (a different value for each field) the scalar field $\varphi$ must be in a minimum state of its potential $V$. This fundamental state of 
the self-interacting scalar field has an low-energy distribution given by $T_{\mu \nu}=V\left(\varphi_{0}\right) g_{\mu \nu}$. A particular form of self-interaction of the scalar field $\varphi$ allows the existence of a constant value $V\left(\varphi_{0}\right)$ that is directly related to the mass of the original Higgs proposal. This scalar field has its own mass, the origin of which remains unclear.

Although the concept of mass pervades almost all the analysis involving gravitational interaction, it is an uncomfortable situation and still to this day there has been no successful attempt to derive a mechanism through which mass is understood as a direct consequence of a dynamical process depending on gravity [5].

According to the origins of General Relativity, the main idea concerning inertia in the realm of gravity goes in the opposite direction to the mechanism that we analyzed in the previous section in the territory of highenergy physics. Indeed, while the Higgs mechanism explores the reduction of a global symmetry into a local one, Mach's principle suggests a cosmical dependence of local properties, making the origin of the mass of a given body dependent on the structure of the whole universe. In this way, there ought to exist a mechanism by means of which this quantity (mass) depends on the state of the Universe. In precedent sections, we described a gravitational mechanism and have shown how this vague idea can achieve a quantitative scheme.

\section{Mass for the Higgs boson}

The procedure for giving mass to bosons in our mechanism follows the same lines. We synthesize its main steps. We start from the Lagrangian

$$
\mathbb{L}=\frac{1}{\kappa} R+\frac{1}{2} W(\varphi) \partial_{\alpha} \varphi \partial^{\alpha} \varphi+B(\varphi) R-\frac{1}{\kappa} \Lambda,
$$

According to [3], we choose

$$
B=-\frac{\beta}{4} \varphi^{2}
$$

and

$$
W=\frac{2 q-6\left(B^{\prime}\right)^{2}}{\alpha_{0}+2 B}
$$

This dynamic represents a scalar field coupled nonminimally with gravity. There is no direct interaction between $\varphi$ and the rest-of-the-universe (ROTU), except through the intermediary of gravity described by a cosmological constant $\Lambda$. Thus $\Lambda$ represents the whole influence of the ROTU on $\varphi$. The dynamics of the scalar field becomes

$$
\square \varphi+\mu^{2} \varphi=0
$$

where

$$
\mu^{2}=\beta \Lambda
$$

where, due to dimensionality arguments, we set $q=1 / \kappa$.

Thus, as a result of the gravitational interaction, the scalar field acquires a mass $\mu$ that depends on the constant $\beta$ and on the existence of $\Lambda$. If $\Lambda$ vanishes then the mass of the field vanishes. The net effect of the nonminimal coupling of gravity with the scalar field corresponds to a specific self-interaction of the scalar field.

\section{COMBINED MACH AND HIGGS MECHANISMS TO GENERATE MASS}

From what we have been presenting it follows that the two mechanisms to generate mass do not exclude each other, but instead may be cooperative. Thus we set the complete Lagrangian as

$$
\begin{aligned}
\mathbb{L} & =\mathbb{L}_{D}+\frac{1}{2} \partial_{\mu} \varphi \partial^{\mu} \varphi-V(\varphi) \\
& -\xi \varphi \bar{\Psi} \\
& -\frac{6}{\kappa}\left(\partial_{\mu} \eta \partial^{\mu} \eta-\frac{1}{6} R \eta^{2}\right) \\
& -\frac{\Lambda}{\kappa}
\end{aligned}
$$

where we distinguish the pure Higgs term qand the pure Mach term and as a consequence of gravitational interaction a mixed one appears. Following the same approach applied in previous cases, we can obtain the formula of mass that we shall analyze afterwards.

\section{General mass formula}

For an arbitrary Higgs potential, the combined mechanism yields the mass formula

$$
M c^{2}=\frac{\sigma \Lambda}{k}+\frac{\sigma V\left(\phi_{0}\right)}{\hbar c}+\hbar c \xi \phi_{0} .
$$

Following the same procedure as in previous analysis and using the standard Higgs potential, the unified MachHiggs mechanism yields the corresponding expression for the mass

$$
M c^{2}=\frac{\sigma \Lambda}{k}-\frac{\sigma}{2} \frac{m_{H}^{2} c^{4}}{(\hbar c)^{3}} \nu^{4}+\xi \nu,
$$

where $\nu=\hbar c \phi_{0}$.

To understand how the mass formula depends on each term we use some values for the constants collected from Particle Data Group ([] $]$ ), to wit:

- Higgs mass range: $125 \mathrm{GeV}<m_{H} c^{2}<180 \mathrm{GeV}$;

- Cosmological constant $(\Lambda): 1,34 \times 10^{-56} \mathrm{~cm}^{-2}$;

- Einstein constant $(k): 168,56 \times 10^{-39} \hbar c(G e V)^{-2}$; 
- Vacuum state of Higgs ( $\nu): 246 \mathrm{GeV}$.

Inserting these numbers into Eq. (20), we obtain

$$
\begin{aligned}
M c^{2} \approx & \sigma\left(0,3 \times 10^{-2} \Lambda \mathrm{cm}^{-1}-6,7 \times 10^{-6} \mathrm{~cm}^{-3}\right) \\
& \times 10^{56} \mathrm{MeV}+246 \xi \mathrm{GeV} .
\end{aligned}
$$

There are several possibilities in this unified mechanism. To exemplify these possibilities we choose the electron mass $\left(M_{e} \approx 0.5 \mathrm{MeV}\right)$ to use in the calculations below. Then we list how $M_{e}$ depends on $\sigma, \xi$ and $\Lambda$ :

- If $\sigma=0$, the gravitational field does not play any role in the mechanism and the only surviving term is the interaction between Higgs and fermions (spontaneous symmetry breaking);

- If the mechanism is dominated by Higgs-fermion interaction, then $\xi \sim 10^{-6}$ and $\sqrt[3]{\sigma}<10^{-17} \mathrm{~cm}$. Note that $\sigma$ is smaller than the electron Compton wavelength $\left(\lambda_{e} \approx 2.4 \times 10^{-14} \mathrm{~cm}\right)$;

- If $\xi=0$ and $\Lambda$ assumes the cosmological value, then its contribution is very small and $\sqrt[3]{\sigma} \sim 10^{-17} \mathrm{~cm}$;

- Whether there is no spontaneous symmetry breaking or in the absence of the Higgs boson, the unique mechanism is the gravitational one. Besides, if $\Lambda$ assumes the cosmological constant value, then $\sqrt[3]{\sigma} \sim 5 \mathrm{~cm}$

- In the case the value of $\Lambda$ is the order of $10^{-3} \mathrm{~cm}^{-2}$, then the three parts can significantly contribute for the electron mass ${ }^{1}$.

\section{CONCLUSION: FROM MACH PRINCIPLE TO THE NEW GRAVITY MECHANISM}

Although a widespread formulation-identified as Mach's principle - that the mass of a body may depend on the overall properties of the rest-of-the-universe and consequently to gravity, the association of this dependence to the smallness of gravitational phenomena was at the origin of the general attitude of disregarding any possibility to attribute to gravity an important role in the generation of mass for all bodies. However, this is nothing but an apparent difficulty and can be eliminated by two steps:

- A direct coupling of matter to the curvature of space-time;

\footnotetext{
1 There is not any compelling reason to identify this constant with the actual cosmological constant or the value of the critical density $10^{-48} \mathrm{Gev}^{4}$ provided by cosmology.
}

- The existence of a vacuum distribution or cosmological constant $\Lambda$.

This idea provides a reliable mechanism by means of which gravity is presented as truly responsible for the generation of the mass. As a result of such a procedure, the final expression of mass depends neither on the intensity nor on the specific properties of the gravitational field. This circumvents all previous criticism against the major role of gravity in the origin of mass.

The model uses a slight modification of Mach's principle. Let us remind that, following Einstein [7], we can understand by this principle the statement according to which the entire inertia of a massive body is the effect of the presence of all other masses, deriving from a kind of interaction with the latter or, in other words, the inertial properties of a body $\mathbb{A}$ are determined by the energy throughout all space. The simplest way to implement this idea is to consider the state that takes into account the whole contribution of the rest-of-the-universe onto $\mathbb{A}$ as the most homogeneous one. Thus, it is natural to relate it to what Einstein attributed to the cosmological constant or, in modern language, the vacuum of all remaining bodies. This means describing the energymomentum distribution of all complementary bodies of $\mathbb{A}$ in the Universe under the form

$$
T_{\mu \nu}(U)=\frac{\Lambda}{\kappa} g_{\mu \nu} .
$$

Note that this distribution of the energy content of the environment of the body $\mathbb{A}$ is similar to the Higgs case, although there is an important distinction concerning the role of this homogeneous distribution of energy on the generation of mass mechanism. As we pointed out above, Higgs' proposal does not go further to explore the consequences of this distribution of energy, since it is not followed by the analysis relating such energy to gravitational processes. Besides, the Higgs mechanism has a very crucial drawback: it is obliged to assume that the mass of the Higgs boson has a different origin than all other particles. On the other hand, within our proposal there is a unique and the same universal origin for the mass of all existing body. Thus, the question "what is the origin of the mass of the Higgs boson?" has the same answer as for all other particles, that is: the inertia of any particle is provided by all other particles mediated by gravity that acts as a catalyst in the fundamental vacuum - represented by the energy distribution $T_{\mu \nu}=\Lambda g_{\mu \nu}$ - and as such provides the corresponding mass. Let us make a final remark that it is possible to understand the Mach principle in a broad sense. Indeed, for the method of obtaining mass using the gravity mechanism, the notion of rest-of-the-universe must be understood as the domain of influence on a given body. As a consequence of this, when we deal with the vacuum represented by the distribution of energy by $T_{\mu \nu}=\Lambda g_{\mu \nu}$ it is completely irrelevant - for the gravitational mechanism of providing mass - whether parameter $\Lambda$ has a classical 
global origin (the Universe) identified with the cosmological constant introduced by Einstein or a restricted one (the environment) identified - as it is the case in the Higgs procedure - to the vacuum of quantum fields.

\section{Acknowledgements}

The major part of this work was done when visiting the International Center for Relativistic Astrophysics
(ICRANet) at Pescara (Italy). We thank professor E. Elbaz and our colleagues of ICRA-Brasil and particularly Dr J.M. Salim for their comments in a previous version of this paper. This work was partially supported by Conselho Nacional de Desenvolvimento Científico e Tecnológico (CNPq), FINEP, and Fundação de Amparo à Pesquisa do Estado de Rio de Janeiro (FAPERJ) of Brazil.

[5] J. V. Narlikar, An Introduction to Cosmology, Cambridge University Press, Cambridge, England (2002). In this book one can find further references to previous attempts and in particular the beautiful proposal of Hoyle and Narlikar.

[6] K. Nakamura et al., Particle Data Group, J. Phys G 37 075021 (2010)

[7] A. Einstein, The Meaning of Relativity, Princeton University Press, Princeton (1950). Z. Naturforsch., 14441 (1959) and earlier papers quoted there. netic Interaction, CERN-report (1971); W. Heisenberg,

for a historical review.

R. H. Dicke, Mach's Principle and a Relativistic Theory Breach Publishers, Les Houches (1964).

[3] M. Novello, Class. Quant. Gravity 28035003 (2011).

[4] B. Zumino, Yang-Mills Theory of Weak and Electromag- 